\title{
STABLE POSITIVE DEFINITE FUNCTIONS
}

\author{
BY
}

\author{
K. R. PARTHASARATHY AND K. $\operatorname{SCHMIDT}\left({ }^{1}\right)$
}

ABSTRACT. This paper investigates the stability of positive definite functions on locally compact groups under one parameter groups of automorphisms. As an application of this it is shown that the only probability distributions on $R^{n}$ which are stable under the full automorphism group $\operatorname{GL}(n, R)$ of $R^{n}$ are the nondegenerate Gaussian distributions. It is furthermore shown that there are no nondegenerate probability distributions of $R^{n}$ which are stable under $\operatorname{SL}(n, R)$.

1. Introduction. P. Levy [1] introduced the notion of a stable probability distribution on the real line and obtained a representation for the Fourier transform of such distributions. As a natural generalization of this idea one can introduce the concept of a stable positive definite function on a general locally compact group $G$. The stability is always with respect to a suitable group of automorphisms of $G$. We are far from obtaining a general representation theorem for this case. The main aim of this article is to obtain a representation of positive definite functions on $G$ which are stable under a one parameter group of automorphisms. As a corollary we are able to show that the only probability distributions on $R^{n}$ which are stable under the full automorphism group $\operatorname{GL}(n, R)$ are Gaussian, and that there are no probability distributions on $R^{n}$ which are stable under the group $\operatorname{SL}(n, R)$ of all $n \times n$ matrices with determinant 1 .

2. Stable positive definite functions. Let $G$ be a locally compact second countable group with identity $e$. We start with a few definitions.

Definition 2.1. A continuous complex valued function $\phi$ on $G$ with $\phi(g)=\overline{\phi\left(g^{-1}\right)}$ for every $g \in G$ is called positive definite if

(1) $\phi(e)=1$,

(2) for any $n \geqslant 1, c_{1}, \cdots, c_{n} \in C$ (the complex numbers) and any $g_{1}, \cdots, g_{n} \in G$, we have

$$
\sum_{i, j=1}^{n} c_{i} \bar{c} \phi_{j} \phi\left(g_{j}^{-1} g_{i}\right) \geqslant 0
$$

Received by the editors November 15, 1972 and, in revised form, December 27, 1973. AMS (MOS) subject classifications (1970). Primary 60B15, 43A35; Secondary 60E05.

(1) Part of this work was done when the authors were visitors at the Mathematics Institute, University of Warwick, Coventry. 
$\phi$ is called conditionally positive definite if

(1') $\phi(e)=0$,

(2') (2.1) holds whenever $\sum_{i=1}^{n} c_{i}=0$.

DEFINITION 2.2. A positive definite function $\phi$ on $G$ is called infinitely divisible if, for any $n \geqslant 1$, there exists a positive definite function $\phi_{n}$ and a continuous homomorphism $\chi_{n}$ from $G$ into the group $T$ of complex numbers of modulus 1 such that $\phi=\chi_{n} \cdot \phi_{n}^{n}$.

The following lemma establishes a close connection between infinitely divisible positive definite functions and conditionally positive definite functions:

Lemma 2.3. Let $G$ be connected and locally connected. A positive definite function $\phi$ on $G$ is infinitely divisible if and only if it is of the form

$$
\phi=\exp \psi
$$

where $\psi$ is a conditionally positive definite function on $G . \psi$ is determined uniquely by (2.2).

This is proved in [2, Theorem 12.9].

In classical probability theory, a positive definite function $\phi$ on the real line $R$ is called stable if for any two positive real numbers $c_{1}, c_{2}$ there exists a positive real number $c_{3}$ and a character $\chi$ of $R$ such that

$$
\phi\left(c_{1} t\right) \cdot \phi\left(c_{2} t\right)=\chi(t) \cdot \phi\left(c_{3} t\right)
$$

for every $t \in R$. Obviously $\phi$ must be infinitely divisible if it satisfies (2.3). A natural generalization of this notion of stability is the following:

DEFINITION 2.4. Let $G$ be a locally compact second countable group and let $\boldsymbol{A}$ be a locally compact second countable group of automorphisms of $\boldsymbol{G}$ such that the map $(\alpha, g) \rightarrow \alpha g, \alpha \in A, g \in G$, is continuous. A positive definite function $\phi$ on $G$ is called stable under $A$ if for any $\alpha_{1}, \alpha_{2} \in A$ there exists an $\alpha_{3} \in A$ and a continuous homomorphism from $G$ to $T$ such that

$$
\phi\left(\alpha_{1} g\right) \cdot \phi\left(\alpha_{2} g\right)=\chi(g) \cdot \phi\left(\alpha_{3} g\right)
$$

for every $g \in G$.

It is again obvious that any $\phi$ which is stable under $A$ must also be infinitely divisible. In particular we have:

LEMMA 2.5. Let $G$ be connected and locally connected, and let $\phi$ be a positive definite function on $G$ which is stable under $A$. Let $\psi$ be the conditionally positive definite function on $G$ satisfying

$$
\phi=\exp \psi .
$$


Put

$$
K(g, h)=\psi\left(h^{-1} g\right)-\psi\left(h^{-1}\right)-\psi(g)
$$

for every $g, h \in G$. Then the following is satisfied: For any $\alpha_{1}, \alpha_{2} \in A$ there exists an $\alpha_{3} \in A$ such that

$$
K\left(\alpha_{1} g, \alpha_{1} h\right)+K\left(\alpha_{2} g, \alpha_{2} h\right)=K\left(\alpha_{3} g, \alpha_{3} h\right)
$$

for every $g, h \in G$.

Proof. The existence of $\psi$ satisfying (2.5) follows from Lemma 2.3. To prove (2.7) we note that, by (2.3),

$$
\exp \left(K\left(\alpha_{1} g, \alpha_{1} h\right)+K\left(\alpha_{2} g, \alpha_{2} h\right)\right)=\exp K\left(\alpha_{3} g, \alpha_{3} h\right)
$$

for every $g, h \in G$. Since $K$ is continuous and since $K(e, e)=0$, the connectedness of $G \times G$ implies (2.7). The lemma is proved.

Lemma 2.6. Let $G, A$ and $K$ be given as in Lemma 2.5. Then the following hold:

(1) For every positive rational number $r$ there exists an element $\alpha(r) \in A$ such that

$$
K(\alpha(r) g, \alpha(r) h)=r \cdot K(g, h)
$$

for every $g, h \in G$.

(2) The set $B$ of all $\beta \in A$ with the property that there exists a constant $c(\beta)$ with

$$
K(\beta g, \beta h)=c(\beta) \cdot K(g, h), \quad g, h \in G,
$$

is a closed subgroup of $A$.

Proof. Putting $\alpha_{1}=\alpha_{2}=I$ (the identity element in $A$ ) we see the existence of $\alpha(2)$ from (2.7). Next we put $\alpha_{1}=\alpha(2), \alpha_{2}=I$, and get $\alpha(3)$. In this manner we can define $\alpha(n)$ for every $n=1,2, \cdots$. Putting $\alpha(1 / n)=$ $\alpha(n)^{-1}$ and $\alpha(p / q)=\alpha(1 / q)^{p}$ for every positive rational number $p / q$ we can assign an $\alpha(r)$ to every rational $r>0$, even though the choice will in general not be unique. The second part of the lemma follows from the fact that $B$ coincides with the set of all $\alpha \in A$ for which the map

$$
(g, h) \rightarrow K(\alpha g, \alpha h) / K(g, h)
$$

from $\{(g, h): g, h \in G, K(g, h) \neq 0\}$ to $C$ is constant in $g, h$. The lemma is proved.

REMARK 2.7. The map $\beta \rightarrow c(\beta)$ defined by (2.9) is a homomorphism from $B$ into the multiplicative group of positive real numbers. If, in particular, 
$A=R$, then $B=A$ and the map $c(\cdot)$ is of the form $c(t)=\exp c_{1} t$ for some real constant $c_{1} \neq 0$.

3. Positive definite functions on $R^{\nu}$ which are stable under $R$. Throughout this section we shall assume $G$ to be a finite dimensional real vector space $R^{\nu}, \nu \geqslant 1$, and $A=\left\{\alpha_{t}, t \in R\right\}$ a continuous 1-parameter group of matrices on $R^{\nu}$. For each $t \in R$ we denote by $\alpha_{t}^{*}$ the adjoint matrix of $\alpha_{t}$, and put $\beta_{t}=\alpha_{-t}^{*}$. Then $\left\{\beta_{t}, t \in R\right\}$ is again a continuous 1-parameter group of automorphisms of $R^{\nu}$.

Definition 3.1. We call the action $(t, v) \rightarrow \alpha_{t} v, t \in R, v \in R^{\nu}$, smooth, if the action $(t, v) \rightarrow \beta_{t} v, t \in R, v \in R^{\nu}$, has the following property: There exists an analytic subset $S$ of $R^{\nu}$ which intersects each orbit in exactly one point.

Let us now choose and fix a 1-parameter group $A=\left\{\alpha_{t}, t \in R\right\}$ which acts smoothly on $R^{\nu}$, and let $\phi$ be a positive definite function on $R^{\nu}$ which is stable under $A$. As remarked in Lemma $2.5, \phi$ is infinitely divisible. We put

$$
\phi=\exp \psi
$$

where $\psi$ is the conditionally positive definite function given in Lemma 2.3 , and write

$$
K\left(v_{1}, v_{2}\right)=\psi\left(v_{1}-v_{2}\right)-\psi\left(v_{1}\right)-\psi\left(-v_{2}\right), \quad v_{1}, v_{2} \in R^{\nu} .
$$

The classical Levy-Khinchine formula on $R^{\nu}$ (see, for example, $[3, \S 16]$ ) tells us that

$$
\begin{aligned}
\psi(v)= & -1 / 2\langle P v, v\rangle+i\langle v, a\rangle \\
& +\int_{R^{\nu}-\{0\}}\left(\exp i\langle v, w\rangle-1-i \frac{\langle v, w\rangle}{1+\|w\|^{2}}\right) d F(w),
\end{aligned}
$$

where $P$ is a positive semidefinite matrix on $R^{\nu},\langle\cdot, \cdot\rangle$ denotes the inner product on $R^{\nu},\|w\|^{2}=\langle w, w\rangle, a$ is a fixed element in $R^{\nu}$, and where $F$ is a $\sigma$-finite measure on $R^{\nu}-\{0\}$ such that

$$
\int(1-\cos \langle v, w\rangle) d F(w)<\infty
$$

for every $v \in R^{\nu}$. $a, P$ and $F$ are all uniquely determined by formula (3.3). Conversely, if $a, P$ and $F$ are given as above and if $F$ satisfies (3.4), then the function $\psi$ defined by (3.3) exists and is conditionally positive definite. Substituting (3.3) into (3.2) we have

$$
\begin{aligned}
K\left(v_{1}, v_{2}\right)= & \left\langle P v_{1}, v_{2}\right\rangle \\
& +\int_{R} \nu-\{0\}\left(\exp i\left\langle v_{1}-v_{2}, w\right\rangle-\exp i\left\langle v_{1}, w\right\rangle\right. \\
& \left.\quad-\exp i\left\langle-v_{2}, w\right\rangle+1\right) d F(w) .
\end{aligned}
$$


LEMMA 3.2. There exists a continuous homomorphism $t \rightarrow \exp c t$ from $R$ to $R^{+}$, the multiplicative group of positive real numbers, satisfying

$$
K\left(\alpha_{t} v, \alpha_{t} w\right)=\exp c t K(v, w)
$$

for every $v, w \in R^{\nu}$. Hence the measure $F$ given in (3.3) satisfies

$$
\exp \operatorname{ct} F\left(\beta_{t}^{-1}\right)=F
$$

for every $t \in R$. If $c \neq 2$, then $P$ must be zero.

Proof. The existence of the homomorphism $t \rightarrow \exp c t$ was pointed out in Corollary 2.7. (3.7) and the conditions on $P$ both follow from (3.5) and (3.6). The lemma is proved.

LEMMA 3.3. Let $S$ be an analytic subset of $R^{\nu}$ which intersects each orbit of $\left\{\beta_{t}, t \in R\right\}$ in exactly one point. Then there exists a $\sigma$-finite measure $\mu$ on $S-\{0\}$ such that

$$
\begin{aligned}
& \int\left(\exp i\langle v, w\rangle-1-i \frac{\langle v, w\rangle}{1+\|w\|^{2}}\right) d F(w) \\
& \quad=\int_{S-\{0\}} \int_{R} \exp c t\left(\exp i\left\langle v, \beta_{t} s\right\rangle-1-i \frac{\left\langle v, \beta_{t} s\right\rangle}{1+\left\|\beta_{t} s\right\|^{2}}\right) d t d \mu(s) .
\end{aligned}
$$

Proof. $F$ is quasi-invariant under $\left\{\beta_{t}, t \in R\right\}$, by (3.7). Applying standard decomposition theory we decompose $F$ into $\sigma$-finite measures which are ergodic under $\left\{\beta_{t}, t \in R\right\}$. Under our assumptions every ergodic measure is supported by a single orbit of $\left\{\beta_{t}, t \in R\right\}$. We can thus write

$$
\begin{aligned}
\int_{R} \nu_{-\{0\}}\left(\exp i\left\langle v_{1}-v_{2}, w\right\rangle-\exp i\left\langle v_{1}, w\right\rangle-\exp i\left\langle-v_{2}, w\right\rangle+1\right) d F(w) \\
=\int_{S-\{0\}}\left(\exp i\left\langle v_{1}-v_{2}, w\right\rangle-\exp i\left\langle v_{1}, w\right\rangle\right. \\
\left.-\exp i\left\langle-v_{2}, w\right\rangle+1\right) d F_{s}(w) d \tilde{\mu}(s),
\end{aligned}
$$

where each $F_{S}$ is a $\sigma$-finite measure concentrated on the orbit $\left\{\beta_{t} s, t \in R\right\}$ in $R^{\nu}-\{0\}$, and where $\tilde{\mu}$ is a probability measure on $S-\{0\}$. From (3.6) and (3.7) we see that, for any $t \in R, s \in S-\{0\}$,

$$
c(t) \cdot F_{s}\left(\beta_{-t}\right)=F_{s} .
$$

This implies the following formula: If $E$ is any Borel subset of $R^{\nu}-\{0\}$ and $s \in S-\{0\}$, then 


$$
F_{s}(E)=f(s) \int_{E \cap\left\{\beta_{t} s, t \in R\right\}} c(t) d t,
$$

where $f(s)$ is a nonnegative constant, and where the map $s \rightarrow f(s), s \in S-\{0\}$, is $\tilde{\mu}$-measurable. We put $d \mu(s)=f(s) d \tilde{\mu}(s)$ and conclude (3.8) from the remarks about the Levy-Khinchine formula preceding Lemma 3.2. The proof is complete.

Combining these results in form of a theorem we get

THEOREM 3.4. Let $\left\{\alpha_{t}, t \in R\right\}$ be a continuous 1-parameter group of $\nu \times \nu$ matrices. Put $\beta_{t}=\alpha_{-t}^{*}$ for every $t \in R$, and assume that there exists an analytic set $S$ in $R^{\nu}$ which intersects each orbit of $\left\{\beta_{t}, t \in R\right\}$ in $R^{\nu}$ in exactly one point. Let $\phi$ be a positive definite function on $R^{\nu}$ which is stable under $\left\{\alpha_{t}, t \in R\right\}$. Then $\phi$ is of the form

$$
\phi=\exp \psi,
$$

where $\psi$ is a conditionally positive definite function on $R^{\nu}$. Furthermore there exists a nonzero real constant $\gamma$, an element $a \in R^{\nu}, a$-finite measure $\mu$ on $S-\{0\}$, and a positive semidefinite $\nu \times \nu$ matrix $P$ such that, for any $v \in R^{\nu}$,

$$
\psi(v)=-1 / 2\langle P v, v\rangle+i \cdot\langle v, a\rangle
$$

$$
+\int_{S-\{0\}} \int_{R}\left(\exp i\left\langle v, \beta_{t} s\right\rangle--1-i \frac{\left\langle v, \beta_{t} s\right\rangle}{1+\left\|\beta_{t} s\right\|^{2}}\right) \cdot \exp \gamma t d t d \mu(s) .
$$

If $\gamma \neq \pm 2$, then $P$ vanishes.

Conversely, any nonzero constant $\gamma \in R$, any $a \in R^{\nu}$, any $\sigma$-finite measure $\mu$ on $S-\{0\}$, and - if $|\gamma|=2-$ any positive semidefinite $\nu \times \nu$ matrix $P$ together define a positive definite function $\phi$ on $R$ which is given by (3.11) and (3.12) and which is stable under $\left\{\alpha_{t}, t \in R\right\}$, if only

$$
\int_{S-\{0\}} \int_{R}\left(1-\cos \left\langle v, \beta_{t} s\right\rangle\right) \cdot \exp \gamma t d t d \mu(s)<\infty
$$

for every $v \in R^{v}$.

Proof. The first part of the theorem is already contained in Lemmas 3.2 and 3.3. The converse follows immediately from (3.4)* In particular we can choose $A$ to be the 1-parameter group $\left\{e^{t} I, t \in R\right\}$, where $I$ is the identity matrix on $R^{\nu}$. An application of Theorem 3.4 together with a well-known computation (see e.g. [2]) leads to the following classical result:

THEOREM 3.5. Let $\phi$ be any positive definite function on $R^{\nu}$ which is stable under $A=\left\{e^{t} I, t \in R\right\}$. Then we have $\phi=\exp \psi$ where $\psi$ is a conditionally positive definite function on $R^{\nu}$ given by any of the following three expressions: 
(1) $\psi(v)=i\langle v, a\rangle-1 / 2\langle P v, v\rangle, \quad v \in R^{\nu}$,

where $a \in R^{\nu}$ and $P$ is a nonnegative definite $\nu \times \nu$ matrix,

(2) $\psi(v)=i\langle u, a\rangle+\operatorname{sgn}(\gamma-1)$

$$
\text { - } \int \exp (-1 / 2 i \pi \gamma \operatorname{sgn}(v, w))|\langle v, w\rangle|^{\gamma} d \mu(w), \quad v \in R^{\nu},
$$

where $a \in R^{\nu}, \gamma$ is a constant satisfying either $0<\gamma<1$ or $1<\gamma<2$, and $\mu$ is a totally finite measure concentrated on $\{w:\|w\|=1\}$ in $R^{\nu}$. (The symbol sgn stands for "sign".)

(3) $\psi(v)=i\langle v, a\rangle-\int \frac{\pi}{2}|\langle v, w\rangle|+i\langle v, w\rangle \log |\langle v, w\rangle| d \mu(w), v \in R^{\nu}$,

where $a \in R^{\nu}$ and where $\mu$ is a totally finite measure on $\{w:\|w\|=1\}$. Conversely, any function $\phi=\exp \psi$ with $\psi$ given by any of the formulas (1)-(3) above will be a positive definite function on $R^{\nu}$ which is stable under $A$.

4. Stable positive definite functions on a type I group. In this section we shall give a brief account of the analysis of positive definite functions on a type I group which are stable under a suitable 1-parameter group of automorphisms. The results are similar to the ones in the abelian case, and since the methods are quite well known and involve little more than the usual measure theoretic techniques used in decomposition theory we shall not include any proofs. Our assumptions are the following:

Let $G$ be a locally compact second countable type I group which is connected and locally connected. We denote by $\hat{G}$ the dual of $G$, i.e. the set of all unitary equivalence classes of continuous irreducible representations of $G$ in a Hilbert space, furnished with the Mackey-Borel structure. If $A=\left\{\alpha_{t}, t \in R\right\}$ is a continuous 1-parameter group of continuous automorphisms of $G$, we can define the adjoint $\left\{\beta_{t}, t \in R\right\}$ as the 1-parameter group of Borel transformations of $\hat{G}$ given by $\beta_{t}=\alpha_{-t}^{*}, t \in R$. Here $\alpha_{t}^{*}$ denotes the map which sends any element $x \in \hat{G}$ containing a representation $g \rightarrow V_{g}$ of $G$ to the element $\alpha_{t}^{*} x$ containing $g \rightarrow V_{\alpha_{t} g}$. Again we assume that there exists an analytic subset $S$ of $\hat{G}$ which intersects each orbit of $\left\{\beta_{t}, t \in R\right\}$ in $\hat{G}$ in exactly one point. Under these assumptions we have

THEOREM 4.1. Let $\phi$ be a positive definite function on $G$ which is stable under $A$. Then there exists a conditionally positive definite function $\psi$ on $G$ with $\phi=\exp \psi$. Furthermore there exist a nonzero real constant $\gamma$, a standard Borel space $X$, a probability measure $\mu$ on $X$, a Borel subset $Y$ of $X, \mu$ measurable families $\left\{H^{x}, x \in X\right\}$ of complex separable Hilbert spaces, $\left\{V^{x}\right.$, $x \in X\}$ of continuous unitary irreducible representations $V^{x}$ of $G$ in $H^{x}$, and $\{\delta(x, \cdot), x \in X\}$ of continuous maps $\delta(x, \cdot): G \rightarrow H^{x}$, which satisfy: 
(1) $\{\delta(x, g), g \in G\}$ spans $H^{x}$ for every $x \in X$.

(2) $V_{g}^{x} \delta(x, h)-\delta(x, g h)+\delta(x, g)=0$ for every $g, h \in G, x \in X$.

(3) $\delta\left(\alpha_{t}(g)\right)=\exp \gamma t \delta(x, g)$ for every $t \in R, g \in G, x \in X-Y$.

(4) $\psi\left(h^{-1} g\right)-\psi\left(h^{-1}\right)-\psi(g)=\int_{Y} \int_{R}\left\langle\delta\left(x, \alpha_{t}(g)\right), \delta\left(x, \alpha_{t}(h)\right)\right\rangle$

$$
\cdot \exp -\gamma t d t d \mu(x)+\int_{X-Y}\langle\delta(x, g), \delta(x, h)\rangle d \mu(x) \text {. }
$$

The proof makes use of results contained in [3], in particular of the Theorems 12.12 and 13.2 there.

5. Stability under $\operatorname{GL}(\nu, R)$ and a characterization of Gaussian positive definite functions. Let again $G=R^{\nu}$ and let $A$ be a locally compact group of $\nu \times \nu$ matrices. Assume $\phi$ is a positive definite function on $R^{\nu}$ which is stable under $A$. Since $\phi$ is infinitely divisible, we can write it as $\phi=\exp \psi$, where $\psi$ is conditionally positive definite. Again we put

$$
K(u, v)=\psi(u-v)-\psi(u)-\psi(-v), \quad u, v \in R^{\nu} .
$$

Let

$$
A_{0}=\left\{\alpha \in A: K(\alpha u, \alpha v)=K(u, v) \text { for all } u, v \in R^{\nu}\right\} .
$$

Clearly $A_{0}$ is a closed subgroup of $A$.

LEMma 5.1. Let $B$ be a finite subgroup of $A$. Then there exists $a \quad \zeta \in A$ such that $\zeta B \zeta^{-1} \subset A_{0}$.

Proof. By (2.7) there exists a $\zeta \in A$ such that

$$
\sum_{\beta \in B} K(\beta v, \beta w)=K(\zeta v, \zeta w)
$$

for every $v, w \in R$. Hence $K(\zeta \beta v, \zeta \beta w)=K(\zeta v, \zeta w)$ for every $v, w \in R^{\nu}$ and every $\beta \in B$. The lemma is proved.

Lemma 5.2. Assume there exist an element $v_{0}$ in $R^{v}$ and a sequence $\left(\alpha_{n}\right)$ in $A$ such that

(1) $\sup _{n}\left|\operatorname{Re} \psi\left(\alpha_{n} v\right)\right|<\infty$ for every $v \in R^{\nu}$,

$$
\text { (2) } \lim _{n}\left\|\alpha_{n} v_{0}\right\|=\infty \text {. }
$$

Then the set $D=\{v:|\phi(v)|=1\}$ contains a nonzero subspace of $R^{\nu}$.

Proof. By Lemma 2.5 there exists a sequence $\left(\beta_{n}\right)$ in $A$ such that

$$
K\left(\beta_{n} v, \beta_{n} w\right)=\frac{1}{n} K(v, w)
$$


for every $n=1,2, \cdots$ and every $v, w \in R^{\nu}$ ( $K$ is given by (6.1)). In particular,

$$
\operatorname{Re} \psi\left(\beta_{n} v\right)=\frac{1}{n} \operatorname{Re} \psi(v)
$$

for every $n=1,2, \cdots$ and every $v \in R^{\nu}$. We choose a sequence $1 \leqslant k(1) \leqslant$ $k(2) \leqslant \cdots \leqslant k(n) \leqslant \cdots$ of integers with

$$
\lim _{n} k(n)=\infty, \quad \lim _{n}\left\|\beta_{k(n)} \alpha_{n} v_{0}\right\|=\infty .
$$

Let, for any $\epsilon>0, S(\epsilon)$ denote the set $S(\epsilon)=\{v:|\operatorname{Re} \psi(v)| \leqslant \epsilon\}$, and put $B(1)=\{v:\|v\|=1\}$. (5.6) implies that

$$
\lim _{n} \operatorname{Re} \psi\left(\beta_{n} \alpha_{n} v\right)=0
$$

for every $v \in R^{\nu}$. (5.7) and the compactness of $B(1)$ together imply that, for every $\epsilon, \delta>0$, there exists an integer $N(\epsilon, \delta)$ such that, for any $n>N(\epsilon, \delta)$,

$$
\beta_{k(n)} \alpha_{n} B(1) \subset S(\epsilon), \text { and }\left\|\beta_{k(n)} \alpha_{n} v_{0}\right\| \geqslant \delta \text {. }
$$

$\beta_{k(n)} \alpha_{n} B(1)$ is a connected set for every $n \geqslant 1$, and contains 0 and $\beta_{k(n)} \alpha_{n} v_{0}$. We conclude that, for every $n \geqslant N(\epsilon, \delta), \beta_{k(n)} \alpha_{n} B(1)$ contains some element of norm $\delta$. In other words, $S(\epsilon) \cap\{v:\|v\|=\delta\}$ and hence $D \cap\{v:\|v\|=\delta\}$ are nonempty sets for every $\delta>0 . D$ is therefore uncountable. Since $D$ is a closed subgroup of $R^{\nu}, D$ must contain a nontrivial linear subspace of $R^{\nu}$. The lemma is proved.

Lemma 5.3. Let $B$ be a subset of $A$ such that

$$
\sup _{\alpha \in B} \log |\operatorname{Re} \psi(\alpha v)|<\infty
$$

for every $v \in R^{\nu}-\{0\}$. Then either $B$ is relatively compact or $D=$ $\{v:|\phi(v)|=1\}$ contains a nonzero subspace of $R^{\nu}$.

Proof. The subset $B$ is relatively compact if and only if $\{\alpha v, \alpha \in B\}$ is relatively compact in $R^{\nu}-\{0\}$ for every $v \neq 0$. (5.8) implies that $\{\alpha v, \alpha \in B\}$ is bounded away from 0 for every $v \neq 0$. If $B$ is not relatively compact, then there exists at least one $v_{0}$ with $\sup _{\alpha \in B}\left\|\alpha v_{0}\right\|=\infty$. Choosing a sequence $\left(\alpha_{n}\right)$ in $B$ with $\lim _{n}\left\|\alpha_{n} v_{0}\right\|=\infty$ and applying Lemma 5.2 we have proved the lemma.

LEMMA 5.4. Let $B$ be a closed subgroup of $A$ such that

$$
\sup _{\alpha \in B}|\operatorname{Re} \psi(\alpha v)|<\infty
$$

for every $v \in R^{\nu}$. Then either $B$ is compact or $D$ contains a nonzero subspace of $R^{\nu}$. 
Proor. All we have to show is that, if $B$ is not compact, there exists an element $v_{0} \in R^{\nu}$ and a sequence $\left(\alpha_{n}\right)$ in $B$ such that $\lim _{n}\left\|\alpha_{n} v_{0}\right\|=\infty$. Indeed, if no such $v_{0}$ exists, and if $B$ is noncompact, then there exists a sequence $\left(\alpha_{n}\right)$ in $B$ and a $w_{0} \in R^{\nu}$ with $\left\|w_{0}\right\|=1$ and $\lim _{n} \alpha_{n} w_{0}=0$. Since $\sup _{n}\left\|\alpha_{n}^{-1} v\right\|<\infty$ for every $v \in R^{\nu}$ and hence $\sup _{n} \sup _{\|v\|<1}\left\|\alpha_{n}^{-1} v\right\|=c_{0}<\infty$, we get

$$
1=\left\|\alpha_{n}^{-1} \alpha_{n} w_{0}\right\| \leqslant c_{0} \cdot\left\|\alpha_{n} w_{0}\right\| \rightarrow 0
$$

which is impossible. An application of Lemma 5.1 concludes the proof.

LEMMA 5.5. Let $\phi$ be a positive definite function on $R^{\nu}$ which is stable under $A$ and which has the additional property that $D=\{v:|\phi(v)|=1\}=\{0\}$. Then the following holds: For any compact subgroup $B$ of $A$ there exists $a$ $\zeta$ in $A$ such that $\zeta B \zeta^{-1} \subset A_{0}\left(A_{0}\right.$ was defined at the beginning of this section).

Proof. If $\alpha$ is an element in $A$ then we denote by $p_{\alpha}$ the probability measure on $A$ concentrated in $\alpha$. For any $n \geqslant 1$ we choose points $\alpha_{n 1}, \cdots$, $\alpha_{n n}$ in $B$ such that the sequence $\lambda_{n}=n^{-1} \sum_{k=1}^{n} p_{\alpha_{n k}}$ tends weakly to the Haar measure $\lambda$ of $B$. By equation (2.6) we can find a sequence $\left(\zeta_{n}\right)$ in $A$ with

$$
\sum_{k=1}^{n} K\left(\alpha_{n k} v, \alpha_{n k} w\right)=K\left(\zeta_{n} v, \zeta_{n} w\right)
$$

for every $v, w \in R^{\nu}$, where $K$ is defined as in (5.1). Defining $\left(\beta_{n}\right)$ by (5.5) we get, for every $v, w \in R^{\nu}, n=1,2, \cdots$

$$
K\left(\beta_{n} \zeta_{n} v, \beta_{n} \zeta_{n} w\right)=\int K(\alpha v, \alpha w) d \lambda_{n}(\alpha) .
$$

$B$ is compact, and hence $\sup _{\alpha \in B} \log |\operatorname{Re} \psi(\alpha v)|<\infty$ for every $v \neq 0$. This implies that

$$
\sup _{n} \log \left|\operatorname{Re} \psi\left(\beta_{n} \zeta_{n} v\right)\right|<\infty,
$$

for every $v \in R^{\nu}-\{0\}$. We conclude from Lemma 5.3 that $\left(\beta_{n} \zeta_{n}\right)$ has a convergent subsequence $\left(\beta_{n_{k}} \zeta_{n_{k}}\right)$, say, whose limit is $\zeta$. From (5.10) we obtain $K(\zeta v, \zeta w)=\int_{B} K(\alpha v, \alpha w) d \lambda(\alpha)$ and thus, for any $\alpha \in B, K(\zeta \alpha v, \zeta \alpha w)=$ $K(\zeta v, \zeta w)$ for every $v, w \in R^{\nu}$. In other words we have $\zeta B \zeta^{-1} \subset A_{0}$. The lemma is proved.

Even though there is-at least at the moment-little hope of obtaining a complete description of all positive definite functions on $R^{\nu}$ which are stable under an arbitrary group $A$ of automorphisms of $R^{\nu}$, the Lemmas 5.1-5.5 make 
it possible to solve the problem in certain special cases when $A$ has a sufficiently large compact subgroup. As an illustration we choose $A$ to be $\mathrm{GL}(\nu, R)$, the group of all automorphisms of $R^{\nu}$, and determine the stable positive definite functions in this case. The result leads to quite an interesting characterization of the Gaussian probability measures on $R^{\nu}$.

THEOREM 5.6. Let $\phi$ be a positive definite function on $R^{\nu}, \nu \geqslant 2$, which is stable under $\mathrm{GL}(\nu, R)$. Then $\phi$ is either degenerate (i.e. everywhere of modulus 1), or $\phi$ is given by

$$
\phi(v)=\exp (\langle v, a\rangle-1 / 2\langle P v, v\rangle), \quad v \in R^{\nu},
$$

where $a \in R^{\nu}$, and where $P$ is a positive definite $\nu \times \nu$ matrix. Conversely, if $a \in R^{\nu}$ and if $P$ is a positive definite $\nu \times \nu$ matrix, then (5.11) defines $a$ positive definite function on $R^{\nu}$ which is stable under $\operatorname{GL}(\nu, R)$.

Proof. If $\phi$ is stable under $\mathrm{GL}(\nu, R)$ then $|\phi|$ is also stable. We put $D=\{v:|\phi(v)|=1\}$ and $A_{1}=\left\{\alpha \in \mathrm{GL}(\nu, R):|\phi(\alpha v)|=|\phi(v)|\right.$ for all $\left.v \in R^{\nu}\right\}$. The classical inequality

$$
1-\left|\phi\left(v_{1}+v_{2}\right)\right| \leqslant\left(1-\left|\phi\left(v_{1}\right)\right|\right)+\left(1-\left|\phi\left(v_{2}\right)\right|\right) \text { for all } v_{1}, v_{2} \in R^{\nu}
$$

implies that $D$ is a subgroup of $R^{\nu}$. Our first aim is to show that $D$ is either equal to $\{0\}$ or to the whole of $R^{\nu}$. We take $Q$ to be the subgroup of $\mathrm{GL}(\nu, R)$ consisting of all permutations and reflections of the coordinates. Since $Q$ is finite we conclude from Lemma 5.1 that there exists a $\zeta_{1} \in \mathrm{GL}(\nu, R)$ with $\zeta_{1} Q \zeta_{1}^{-1} \subset A_{1}$. Hence $Q$ leaves $\zeta_{1}^{-1} D$ invariant. Let us assume that $D \neq\{0\}$. If $v \neq 0$ is an element of $D$, then $w=\zeta_{1}^{-1} v=\left(w_{1}, \cdots, w_{\nu}\right)$ is nonzero. We assume without loss of generality that $w_{1} \neq 0$. The invariance of the group $\zeta_{1}^{-1} D$ under $Q$ implies that $\left(w_{1},-w_{2}, \cdots,-w_{\nu}\right)$ and $\left(2 w_{1}, 0, \cdots, 0\right)$ are both elements of $\zeta_{1}^{-1} D$. Using again invariance under $Q$ we see that all points of the form $\left(2 k_{1} w_{1}, \cdots, 2 k_{\nu} w_{1}\right)$ are contained in $\zeta_{1}^{-1} D$, where $\left(k_{1}, \cdots, k_{\nu}\right)$ is any $\nu$-tuple of integers. This implies that the quotient $R^{\nu} / \zeta_{1}^{-1} D$ is compact. Since furthermore $\phi$ is infinitely divisible and hence everywhere nonzero, we conclude from the inequality (5.12) that $|\phi|$ is bounded away from zero. Writing $\phi=\exp \psi$, where $\psi$ is conditionally positive definite (see Lemma 2.3) we see that $\operatorname{Re} \psi$ is bounded. Assume now that $\operatorname{Re} \psi\left(v_{0}\right) \neq 0$ for some $v_{0} \in R^{\nu}$, i.e. that $D \neq R^{\nu}$. By Lemma 2.6 we can find an $\alpha(r) \in$ $\mathrm{GL}(\nu, R)$ for every positive rational $r$, such that $\operatorname{Re} \psi\left(\alpha(r) v_{0}\right)=r \cdot \operatorname{Re} \psi\left(v_{0}\right)$, which contradicts the boundedness of $\operatorname{Re} \psi$. In other words: If $D$ is not equal to $\{0\}$ then $D=R^{\nu}$, in which case $\phi$ is degenerate.

Let us therefore assume from now on that $D=\{0\}$, and let us investigate the size of $A_{1}$. It follows from Lemma 5.5 that there exists a $\zeta_{2}$ such that 
$\zeta_{2} O(\nu) \zeta_{2}^{-1} \subset A_{0} \subset A_{1}$, where $O(\nu)$ is the orthogonal group on $R^{\nu}$. In particular, $\widetilde{\phi}(v)=\left|\phi\left(\zeta_{2} v\right)\right|$ is a function of $\|v\|$ alone.

Our next step will be to reduce the problem to one dimension. Choose a fixed orthonormal basis $\left(e_{1}, \cdots, e_{\nu}\right)$ in $R^{\nu}$ and consider the subgroup $\Sigma$ of all matrices in $\operatorname{GL}(\nu, R)$ which are diagonal in this basis and of the form

$$
\left[\begin{array}{rrrrr}
\lambda & & & & \\
& \lambda^{-1} & & & \\
& 1 & & \\
& & \cdot & & \\
& & & \cdot & \\
& & & \cdot & \\
& & & & 1
\end{array}\right], \quad \lambda>0 .
$$

For any $\lambda_{1}, \lambda_{2}>0$ there exists an $\alpha$ in $\operatorname{GL}(\nu, R)$ such that

$$
\tilde{\phi}\left(\lambda_{1} t e_{1}\right) \tilde{\phi}\left(\lambda_{2} t e_{1}\right)=\tilde{\phi}\left(\alpha t e_{1}\right)
$$

for every $t \in R$. Since $\widetilde{\phi}$ is only dependent on the norm of its argument, we can replace $\alpha$ by some element in $\Sigma$. We thus get: For any $\lambda_{1}, \lambda_{2}>0$ there exists a $\lambda_{3}>0$ such that

$$
\widetilde{\phi}\left(\lambda_{1} t e_{1}\right) \tilde{\phi}\left(\lambda_{2} t e_{1}\right)=\widetilde{\phi}\left(\lambda_{3} t e_{1}\right)
$$

for every $t \in R$. Hence $t \rightarrow \widetilde{\phi}\left(t e_{1}\right)$ is a real valued positive definite function on $R$ which is stable under the group $\left\{e^{t}, t \in R\right\}$. Applying Theorem 3.5 we see that $\tilde{\phi}(v)=\exp -c\|v\|^{\gamma}$ for every $v \in R^{\nu}$, where $c$ is a positive constant and where $\gamma$ satisfies $0<\gamma \leqslant 2$.

Let us finally prove that $\gamma=2$. The stability of $\tilde{\phi}$ under $\operatorname{GL}(\nu, R) \mathrm{im}$ plies that for any $\alpha_{1}, \alpha_{2} \in \mathrm{GL}(\nu, R)$ there exists an $\alpha_{3} \in \mathrm{GL}(\nu, R)$ such that

$$
\left\|\alpha_{1} v\right\| \gamma+\left\|\alpha_{2} v\right\| \gamma=\left\|\alpha_{3} v\right\|^{\gamma}
$$

for every $v \in R^{\nu}$. Choosing

$$
\alpha_{1}=\left[\begin{array}{llll}
\lambda & & \\
& \lambda^{-1} & \\
& & \cdot & \\
& & \cdot
\end{array}\right] \in \Sigma,
$$

$\alpha_{2}=I$ (the identity matrix), and $v=x e_{1}+y e_{2}, x, y \in R$, we see that

$$
\left(\lambda x^{2}+\lambda^{-1} y^{2}\right)^{\gamma / 2}+\left(x^{2}+y^{2}\right)^{\gamma / 2}=\left(a_{1} x^{2}+a_{2} x y+a_{3} y^{2}\right)^{\gamma / 2}
$$

for every $x, y \in R$, where $a_{1}, a_{2}, a_{3}$ are real numbers. Differentiating with 
respect to $x$ and putting $x=0$ we see that $a_{2}=0$. We get $(\gamma / 2=\eta)$

$$
\left(\lambda x^{2}+\lambda^{-1} y^{2}\right)^{\eta}+\left(x^{2}+y^{2}\right)^{\eta}=\left(a_{1} x^{2}+a_{3} y^{2}\right)^{\eta}
$$

for every $x, y \in R$, and for every $\lambda>0$. Hence $a_{1}=\left(\lambda^{\eta}+1\right)^{1 / \eta}, a_{3}=$ $\left(\lambda^{-\eta}+1\right)^{1 / \eta}$, and

$$
\left(\lambda x^{2}+\lambda^{-1} y^{2}\right)^{\eta}+\left(x^{2}+y^{2}\right)^{\eta}=\left(\left(\lambda^{n}+1\right)^{1 / \eta} x^{2}+\left(\lambda^{-\eta}+1\right)^{1 / \eta} y^{2}\right)^{\eta}
$$

for every $\lambda>0, x, y \in R$. Putting $y^{2}=\lambda$ we have

$$
\left(\lambda x^{2}+1\right)^{\eta}+\left(x^{2}+\lambda\right)^{\eta}=\left(\lambda^{\eta}+1\right)\left(x^{2}+1\right)^{\eta} .
$$

If we differentiate twice with respect to $x$ and put $x=0$ afterwards we get $(\lambda+1)\left(\lambda^{\eta-1}-1\right)=0$ for every $\lambda>0$, and hence $\gamma / 2=\eta=1$. Thus we have

$$
\widetilde{\phi}(v)=\left|\phi\left(\zeta_{2} v\right)\right|=\exp -c\|v\|^{2}
$$

for every $v \in R^{\nu}$, and for some positive constant $c$. Hence

$$
|\phi(v)|=\exp -1 / 2\|P u\|^{2},
$$

where $P=2 \zeta^{*-1} \zeta^{-1}$. A well-known theorem of probability theory states that any positive definite function $\phi$ whose modulus is of the form (5.13) satisfies (5.11) for some $a \in R^{\nu}$. The theorem is proved, since the converse is obvious.

THEOREM 5.7. There are no nondegenerate positive definite functions on $R^{\nu}$ which are stable under $\operatorname{SL}(\nu, R)$.

Proof. Assume there exists a nondegenerate positive definite function $\phi$ on $R^{\nu}$ which is stable under $\operatorname{SL}(\nu, R)$. We can use the proof of Theorem 5.6 to show that $|\phi(v)|=\exp -1 / 2\|P u\|^{2}$, where $P$ is a positive definite $\nu \times \nu$ matrix (note that all elements of $\operatorname{GL}(\nu, R)$ which were used in the proof of Theorem 5.6 were actually elements of $\operatorname{SL}(\nu, R)$ ). We conclude that, for any $\alpha_{1}, \alpha_{2} \in \operatorname{SL}(\nu, R)$ there exists an $\alpha_{3} \in \mathrm{SL}(\nu, R)$ such that

$$
\left\|\alpha_{1} v\right\|^{2}+\left\|\alpha_{2} v\right\|^{2}=\left\|\alpha_{3} v\right\|^{2}
$$

for every $v \in R^{\nu}$. We put $\alpha_{1}, \alpha_{2}=I$ and see immediately that $\alpha_{3}$ cannot be an element of $\operatorname{SL}(\nu, R)$, which contradicts the earlier assumption that $\phi$ is stable under $\operatorname{SL}(\nu, R)$. The theorem is proved.

REMARK 5.8. To give another application of these methods one might take $G=C$ (the additive group of complex numbers), and $A=C_{0}$, the multiplicative group of nonzero complex numbers, which acts on $C$ by multiplication. Any positive definite function $\phi$ on $C$ which is stable under $C_{0}$ must be rotation invariant as a consequence of Lemma 5.5, if it is not degenerate. Applying once 
again Theorem 3.5 we see that $\phi$ must be given by $\phi(z)=\exp -c|z|^{\gamma}, z \in C$, where $0<\gamma \leqslant 2$, and $c>0$.

\section{REFERENCES}

1. P. Lévy, Théorie de l'addition des variables aléatoires, Gauthier-Villars, Paris, 1954.

2. E. Lukacs, Characteristic functions, Griffin, London, 1970.

3. K. R. Parthasarathy and K. Schmidt, Positive definite kernels, continuous tensor products, and central limit theorems of probability theory, Lecture Notes in Math., vol. 272, Springer-Verlag, Berlin and New York, 1972.

MATHEMATICS DEPARTMENT, UNIVERSITY OF BOMBAY, BOMBAY, INDIA

MATHEMATICS DEPARTMENT, BEDFORD COLLEGE, UNIVERSITY OF LONDON, LONDON, GREAT BRITAIN

Current address (of both authors): Mathematics Institute, University of Warwick, Coventry, Great Britain 